\title{
Measurement of the Branching Fraction for $B^{-} \rightarrow D^{0} K^{*-}$
}

B. Aubert,${ }^{1}$ R. Barate,${ }^{1}$ D. Boutigny,${ }^{1}$ F. Couderc,${ }^{1}$ J.-M. Gaillard, ${ }^{1}$ A. Hicheur,${ }^{1}$ Y. Karyotakis,${ }^{1}$ J. P. Lees,${ }^{1}$ P. Robbe,${ }^{1}$ V. Tisserand,${ }^{1}$ A. Zghiche,${ }^{1}$ A. Palano, ${ }^{2}$ A. Pompili,${ }^{2}$ J. C. Chen,${ }^{3}$ N. D. Qi,${ }^{3}$ G. Rong, ${ }^{3}$ P. Wang,${ }^{3}$ Y. S. Zhu,${ }^{3}$ G. Eigen, ${ }^{4}$ I. Ofte, ${ }^{4}$ B. Stugu, ${ }^{4}$ G. S. Abrams,${ }^{5}$ A. W. Borgland,${ }^{5}$ A. B. Breon,${ }^{5}$ D. N. Brown, ${ }^{5}$ J. Button-Shafer, ${ }^{5}$ R. N. Cahn, ${ }^{5}$ E. Charles,${ }^{5}$ C. T. Day ${ }^{5}$ M. S. Gill, ${ }^{5}$ A. V. Gritsan,${ }^{5}$ Y. Groysman,${ }^{5}$ R. G. Jacobsen, ${ }^{5}$ R. W. Kadel,${ }^{5}$ J. Kadyk,${ }^{5}$ L. T. Kerth, ${ }^{5}$ Yu. G. Kolomensky, ${ }^{5}$ G. Kukartsev, ${ }^{5}$ C. LeClerc,${ }^{5}$ M. E. Levi,${ }^{5}$ G. Lynch,${ }^{5}$ L. M. Mir,${ }^{5}$ P. J. Oddone,${ }^{5}$ T. J. Orimoto,${ }^{5}$ M. Pripstein,${ }^{5}$ N. A. Roe, ${ }^{5}$ A. Romosan,${ }^{5}$ M. T. Ronan, ${ }^{5}$ V. G. Shelkov, ${ }^{5}$ A. V. Telnov, ${ }^{5}$ W. A. Wenzel,${ }^{5}$ K. Ford,${ }^{6}$ T. J. Harrison, ${ }^{6}$ C. M. Hawkes,${ }^{6}$ D. J. Knowles, ${ }^{6}$ S. E. Morgan,${ }^{6}$ R. C. Penny, ${ }^{6}$ A. T. Watson, ${ }^{6}$ N. K. Watson, ${ }^{6}$ K. Goetzen, ${ }^{7}$ T. Held,${ }^{7}$ H. Koch, ${ }^{7}$ B. Lewandowski, ${ }^{7}$ M. Pelizaeus, ${ }^{7}$ K. Peters, ${ }^{7}$ H. Schmuecker, ${ }^{7}$ M. Steinke, ${ }^{7}$ J. T. Boyd,${ }^{8}$ N. Chevalier,${ }^{8}$ W. N. Cottingham, ${ }^{8}$ M. P. Kelly, ${ }^{8}$ T. E. Latham,${ }^{8}$ C. Mackay,${ }^{8}$ F. F. Wilson, ${ }^{8}$ K. Abe,${ }^{9}$ T. Cuhadar-Donszelmann, ${ }^{9}$ C. Hearty,${ }^{9}$ T. S. Mattison, ${ }^{9}$ J. A. McKenna, ${ }^{9}$ D. Thiessen,${ }^{9}$ P. Kyberd,${ }^{10}$ A. K. McKemey, ${ }^{10}$ L. Teodorescu, ${ }^{10}$ V. E. Blinov, ${ }^{11}$ A. D. Bukin, ${ }^{11}$ V. B. Golubev,${ }^{11}$ V. N. Ivanchenko, ${ }^{11}$ E. A. Kravchenko, ${ }^{11}$ A. P. Onuchin, ${ }^{11}$ S. I. Serednyakov ${ }^{11}$ Yu. I. Skovpen, ${ }^{11}$ E. P. Solodov, ${ }^{11}$ A. N. Yushkov, ${ }^{11}$ D. Best,${ }^{12}$ M. Bruinsma, ${ }^{12}$ M. Chao, ${ }^{12}$ D. Kirkby, ${ }^{12}$ A. J. Lankford, ${ }^{12}$ M. Mandelkern,${ }^{12}$ R. K. Mommsen, ${ }^{12}$ W. Roethel,${ }^{12}$ D. P. Stoker,${ }^{12}$ C. Buchanan,${ }^{13}$ B. L. Hartfiel, ${ }^{13}$ J. W. Gary, ${ }^{14}$ J. Layter,${ }^{14}$ B. C. Shen, ${ }^{14}$ K. Wang,${ }^{14}$ D. del Re,${ }^{15}$ H. K. Hadavand,${ }^{15}$ E. J. Hilll ${ }^{15}$ D. B. MacFarlane,${ }^{15}$ H. P. Paar, ${ }^{15}$ Sh. Rahatlou ${ }^{15}$ V. Sharma,${ }^{15}$ J. W. Berryhill,${ }^{16}$ C. Campagnari,${ }^{16}$ B. Dahmes ${ }^{16}$ S. L. Levy, ${ }^{16}$ O. Long, ${ }^{16}$ A. Lu,${ }^{16}$ M. A. Mazur, ${ }^{16}$ J. D. Richman, ${ }^{16}$ W. Verkerke,${ }^{16}$ T. W. Beck,,${ }^{17}$ J. Beringer,${ }^{17}$ A. M. Eisner ${ }^{17}$ C. A. Heusch, ${ }^{17}$ W. S. Lockman, ${ }^{17}$ T. Schalk, ${ }^{17}$ R. E. Schmitz, ${ }^{17}$ B. A. Schumm, ${ }^{17}$ A. Seiden, ${ }^{17}$

P. Spradlin, ${ }^{17}$ M. Turri, ${ }^{17}$ W. Walkowiak, ${ }^{17}$ D. C. Williams, ${ }^{17}$ M. G. Wilson, ${ }^{17}$ J. Albert,${ }^{18}$ E. Chen, ${ }^{18}$

G. P. Dubois-Felsmann, ${ }^{18}$ A. Dvoretskii ${ }^{18}$ R. J. Erwin, ${ }^{18}$ D. G. Hitlin, ${ }^{18}$ I. Narsky, ${ }^{18}$ T. Piatenko, ${ }^{18}$ F. C. Porter, ${ }^{18}$

A. Ryd, ${ }^{18}$ A. Samuel, ${ }^{18}$ S. Yang, ${ }^{18}$ S. Jayatilleke, ${ }^{19}$ G. Mancinelli, ${ }^{19}$ B. T. Meadows ${ }^{19}$ M. D. Sokoloff,${ }^{19}$

T. Abe,${ }^{20}$ F. Blanc,${ }^{20}$ P. Bloom,${ }^{20}$ S. Chen, ${ }^{20}$ P. J. Clark, ${ }^{20}$ W. T. Ford,${ }^{20}$ U. Nauenberg, ${ }^{20}$ A. Olivas, ${ }^{20}$ P. Rankin, ${ }^{20}$ J. Roy ${ }^{20}$ J. G. Smith, ${ }^{20}$ W. C. van Hoek ${ }^{20}$ L. Zhang, ${ }^{20}$ J. L. Harton, ${ }^{21}$ T. Hu, ${ }^{21}$ A. Soffer,${ }^{21}$ W. H. Toki, ${ }^{21}$ R. J. Wilson, ${ }^{21}$ J. Zhang, ${ }^{21}$ D. Altenburg, ${ }^{22}$ T. Brandt,${ }^{22}$ J. Brose,${ }^{22}$ T. Colberg, ${ }^{22}$ M. Dickopp ${ }^{22}$

R. S. Dubitzky, ${ }^{22}$ A. Hauke, ${ }^{22}$ H. M. Lacker, ${ }^{22}$ E. Maly, ${ }^{22}$ R. Müller-Pfefferkorn, ${ }^{22}$ R. Nogowski, ${ }^{22}$ S. Otto, ${ }^{22}$

J. Schubert,${ }^{22}$ K. R. Schubert,${ }^{22}$ R. Schwierz, ${ }^{22}$ B. Spaan, ${ }^{22}$ L. Wilden ${ }^{22}$ D. Bernard, ${ }^{23}$ G. R. Bonneaud ${ }^{23}$ F. Brochard, ${ }^{23}$ J. Cohen-Tanugi, ${ }^{23}$ P. Grenier,${ }^{23}$ Ch. Thiebaux,${ }^{23}$ G. Vasileiadis, ${ }^{23}$ M. Verderi, ${ }^{23}$ A. Khan, ${ }^{24}$ D. Lavin,${ }^{24}$ F. Muheim, ${ }^{24}$ S. Playfer, ${ }^{24}$ J. E. Swain, ${ }^{24}$ M. Andreotti,${ }^{25}$ V. Azzolini, ${ }^{25}$ D. Bettoni, ${ }^{25}$ C. Bozzi ${ }^{25}$

R. Calabrese ${ }^{25}$ G. Cibinetto ${ }^{25}$ E. Luppi,${ }^{25}$ M. Negrini, ${ }^{25}$ L. Piemontese,${ }^{25}$ A. Sarti, ${ }^{25}$ E. Treadwell,,${ }^{26}$

R. Baldini-Ferroli, ${ }^{27}$ A. Calcaterra, ${ }^{27}$ R. de Sangro, ${ }^{27}$ D. Falciai, ${ }^{27}$ G. Finocchiaro ${ }^{27}$ P. Patteri,${ }^{27}$ M. Piccolo, ${ }^{27}$

A. Zallo, ${ }^{27}$ A. Buzzo,${ }^{28}$ R. Capra,${ }^{28}$ R. Contri, ${ }^{28}$ G. Crosetti, ${ }^{28}$ M. Lo Vetere,${ }^{28}$ M. Macri,${ }^{28}$ M. R. Monge,${ }^{28}$

S. Passaggio, ${ }^{28}$ C. Patrignani, ${ }^{28}$ E. Robutti, ${ }^{28}$ A. Santroni, ${ }^{28}$ S. Tosi, ${ }^{28}$ S. Bailey,${ }^{29}$ M. Morii, ${ }^{29}$ E. Won, ${ }^{29}$ W. Bhimji, ${ }^{30}$ D. A. Bowerman, ${ }^{30}$ P. D. Dauncey,${ }^{30}$ U. Egede,${ }^{30}$ I. Eschrich,${ }^{30}$ J. R. Gaillard,${ }^{30}$ G. W. Morton, ${ }^{30}$ J. A. Nash,${ }^{30}$ G. P. Taylor ${ }^{30}$ G. J. Grenier, ${ }^{31}$ S.-J. Lee, ${ }^{31}$ U. Mallik, ${ }^{31}$ J. Cochran,${ }^{32}$ H. B. Crawley ${ }^{32}$ J. Lamsa, ${ }^{32}$ W. T. Meyer ${ }^{32}$ S. Prell, ${ }^{32}$ E. I. Rosenberg, ${ }^{32}$ J. Yi, ${ }^{32}$ M. Davier, ${ }^{33}$ G. Grosdidier, ${ }^{33}$ A. Höcker, ${ }^{33}$ S. Laplace, ${ }^{33}$ F. Le

Diberder, ${ }^{33}$ V. Lepeltier, ${ }^{33}$ A. M. Lutz,${ }^{33}$ T. C. Petersen, ${ }^{33}$ S. Plaszczynski, ${ }^{33}$ M. H. Schune, ${ }^{33}$ L. Tantot ${ }^{33}$

G. Wormser, ${ }^{33}$ V. Brigljević, ${ }^{34}$ C. H. Cheng, ${ }^{34}$ D. J. Lange,${ }^{34}$ M. C. Simani,${ }^{34}$ D. M. Wright,${ }^{34}$ A. J. Bevan, ${ }^{35}$ J. P. Coleman, ${ }^{35}$ J. R. Fry ${ }^{35}$ E. Gabathuler, ${ }^{35}$ R. Gamet, ${ }^{35}$ M. Kay ${ }^{35}$ R. J. Parry ${ }^{35}$ D. J. Payne,${ }^{35}$ R. J. Sloane,${ }^{35}$ C. Touramanis, ${ }^{35}$ J. J. Back ${ }^{36}$ P. F. Harrison, ${ }^{36}$ H. W. Shorthouse, ${ }^{36}$ P. B. Vidal, ${ }^{36}$ C. L. Brown, ${ }^{37}$ G. Cowan, ${ }^{37}$ R. L. Flack ${ }^{37}$ H. U. Flaecher,${ }^{37}$ S. George, ${ }^{37}$ M. G. Green, ${ }^{37}$ A. Kurup,${ }^{37}$ C. E. Marker,${ }^{37}$ T. R. McMahon, ${ }^{37}$ S. Ricciardi, ${ }^{37}$ F. Salvatore, ${ }^{37}$ G. Vaitsas, ${ }^{37}$ M. A. Winter,,${ }^{37}$ D. Brown, ${ }^{38}$ C. L. Davis, ${ }^{38}$ J. Allison, ${ }^{39}$ N. R. Barlow,${ }^{39}$ R. J. Barlow ${ }^{39}$ P. A. Hart, ${ }^{39}$ M. C. Hodgkinson, ${ }^{39}$ F. Jackson, ${ }^{39}$ G. D. Lafferty ${ }^{39}$ A. J. Lyon, ${ }^{39}$ J. H. Weatherall, ${ }^{39}$ J. C. Williams, ${ }^{39}$ A. Farbin, ${ }^{40}$ A. Jawahery, ${ }^{40}$ D. Kovalskyi, ${ }^{40}$ C. K. Lae,${ }^{40}$ V. Lillard,${ }^{40}$ D. A. Roberts, ${ }^{40}$ G. Blaylock, ${ }^{41}$ C. Dallapiccola ${ }^{41}$ K. T. Flood, ${ }^{41}$ S. S. Hertzbach, ${ }^{41}$ R. Kofler, ${ }^{41}$ V. B. Koptchev, ${ }^{41}$ T. B. Moore, ${ }^{41}$ 
S. Saremi, ${ }^{41}$ H. Staengle, ${ }^{41}$ S. Willocq,${ }^{41}$ R. Cowan, ${ }^{42}$ G. Sciolla, ${ }^{42}$ F. Taylor, ${ }^{42}$ R. K. Yamamoto, ${ }^{42}$ D. J. J. Mangeol, ${ }^{43}$ P. M. Patel, ${ }^{43}$ S. H. Robertson, ${ }^{43}$ A. Lazzaro, ${ }^{44}$ F. Palombo, ${ }^{44}$ J. M. Bauer,${ }^{45}$ L. Cremaldi, ${ }^{45}$

V. Eschenburg, ${ }^{45}$ R. Godang, ${ }^{45}$ R. Kroeger, ${ }^{45}$ J. Reidy, ${ }^{45}$ D. A. Sanders, ${ }^{45}$ D. J. Summers,${ }^{45}$ H. W. Zhao, ${ }^{45}$

S. Brunet, ${ }^{46}$ D. Cote-Ahern, ${ }^{46}$ P. Taras, ${ }^{46}$ H. Nicholson, ${ }^{47}$ C. Cartaro, ${ }^{48}$ N. Cavallo, ${ }^{48}$ G. De Nardo, ${ }^{48}$

F. Fabozzi $,{ }^{48},{ }^{*}$ C. Gatto, ${ }^{48}$ L. Lista,${ }^{48}$ P. Paolucci, ${ }^{48}$ D. Piccolo, ${ }^{48}$ C. Sciacca, ${ }^{48}$ M. A. Baak, ${ }^{49}$ G. Raven, ${ }^{49}$

J. M. LoSecco, ${ }^{50}$ T. A. Gabriel, ${ }^{51}$ B. Brau, ${ }^{52}$ K. K. Gan, ${ }^{52}$ K. Honscheid, ${ }^{52}$ D. Hufnagel, ${ }^{2}$ H. Kagan, ${ }^{52}$ R. Kass, ${ }^{52}$

T. Pulliam, ${ }^{52}$ Q. K. Wong, ${ }^{52}$ J. Brau, ${ }^{53}$ R. Frey, ${ }^{53}$ O. Igonkina, ${ }^{53}$ C. T. Potter, ${ }^{53}$ N. B. Sinev, ${ }^{53}$ D. Strom, ${ }^{53}$

E. Torrence, ${ }^{53}$ F. Colecchia, ${ }^{54}$ A. Dorigo, ${ }^{54}$ F. Galeazzi, ${ }^{54}$ M. Margoni, ${ }^{54}$ M. Morandin, ${ }^{54}$ M. Posocco, ${ }^{54}$

M. Rotondo, ${ }^{54}$ F. Simonetto, ${ }^{54}$ R. Stroili, ${ }^{54}$ G. Tiozzo, ${ }^{54}$ C. Voci, ${ }^{54}$ M. Benayoun, ${ }^{55}$ H. Briand, ${ }^{55}$ J. Chauveau, ${ }^{55}$

P. David, ${ }^{55}$ Ch. de la Vaissière, ${ }^{55}$ L. Del Buono, ${ }^{55}$ O. Hamon, ${ }^{55}$ M. J. J. John, ${ }^{55}$ Ph. Leruste, ${ }^{55}$ J. Ocariz, ${ }^{55}$

M. Pivk, ${ }^{55}$ L. Roos, ${ }^{55}$ J. Stark, ${ }^{55}$ S. T'Jampens, ${ }^{55}$ G. Therin, ${ }^{55}$ P. F. Manfredi, ${ }^{56}$ V. Re, ${ }^{56}$ P. K. Behera, ${ }^{57}$

L. Gladney, ${ }^{57}$ Q. H. Guo, ${ }^{57}$ J. Panetta, ${ }^{57}$ F. Anulli, ${ }^{27,}{ }^{58}$ M. Biasini, ${ }^{58}$ I. M. Peruzzi, ${ }^{27,}{ }^{58}$ M. Pioppi, ${ }^{58}$ C. Angelini, ${ }^{59}$

G. Batignani, ${ }^{59}$ S. Bettarini, ${ }^{59}$ M. Bondioli, ${ }^{59}$ F. Bucci, ${ }^{59}$ G. Calderini, ${ }^{59}$ M. Carpinelli, ${ }^{59}$ V. Del Gamba, ${ }^{59}$

F. Forti, ${ }^{59}$ M. A. Giorgi, ${ }^{59}$ A. Lusiani, ${ }^{59}$ G. Marchiori, ${ }^{59}$ F. Martinez-Vidal, ${ }^{59, ~} \dagger$ M. Morganti, ${ }^{59}$ N. Neri, ${ }^{59}$

E. Paoloni, ${ }^{59}$ M. Rama, ${ }^{59}$ G. Rizzo, ${ }^{59}$ F. Sandrelli, ${ }^{59}$ J. Walsh, ${ }^{59}$ M. Haire, ${ }^{60}$ D. Judd, ${ }^{60}$ K. Paick, ${ }^{60}$

D. E. Wagoner, ${ }^{60}$ N. Danielson, ${ }^{61}$ P. Elmer, ${ }^{61}$ C. Lu, ${ }^{61}$ V. Miftakov, ${ }^{61}$ J. Olsen, ${ }^{61}$ A. J. S. Smith, ${ }^{61}$

H. A. Tanaka, ${ }^{61}$ E. W. Varnes ${ }^{61}$ F. Bellini, ${ }^{62}$ G. Cavoto, ${ }^{61,62}$ R. Faccini, ${ }^{62}$ F. Ferrarotto, ${ }^{62}$ F. Ferroni, ${ }^{62}$

M. Gaspero, ${ }^{62}$ M. A. Mazzoni, ${ }^{62}$ S. Morganti, ${ }^{62}$ M. Pierini, ${ }^{62}$ G. Piredda, ${ }^{62}$ F. Safai Tehrani, ${ }^{62}$ C. Voena, ${ }^{62}$

S. Christ, ${ }^{63}$ G. Wagner, ${ }^{63}$ R. Waldi, ${ }^{63}$ T. Adye, ${ }^{64}$ N. De Groot, ${ }^{64}$ B. Franek, ${ }^{64}$ N. I. Geddes, ${ }^{64}$ G. P. Gopal, ${ }^{64}$

E. O. Olaiya, ${ }^{64}$ S. M. Xella, ${ }^{64}$ R. Aleksan, ${ }^{65}$ S. Emery, ${ }^{65}$ A. Gaidot, ${ }^{65}$ S. F. Ganzhur, ${ }^{65}$ P.-F. Giraud, ${ }^{65}$ G. Hamel de

Monchenault, ${ }^{65}$ W. Kozanecki, ${ }^{65}$ M. Langer, ${ }^{65}$ M. Legendre, ${ }^{65}$ G. W. London, ${ }^{65}$ B. Mayer, ${ }^{65}$ G. Schott, ${ }^{65}$

G. Vasseur, ${ }^{65}$ Ch. Yeche ${ }^{65}$ M. Zito, ${ }^{65}$ M. V. Purohit,${ }^{66}$ A. W. Weidemann, ${ }^{66}$ F. X. Yumiceva, ${ }^{66}$ D. Aston, ${ }^{67}$

R. Bartoldus, ${ }^{67}$ N. Berger, ${ }^{67}$ A. M. Boyarski, ${ }^{67}$ O. L. Buchmueller, ${ }^{67}$ M. R. Convery, ${ }^{67}$ M. Cristinziani, ${ }^{67}$ D. Dong, ${ }^{67}$

J. Dorfan, ${ }^{67}$ D. Dujmic, ${ }^{67}$ W. Dunwoodie, ${ }^{67}$ E. E. Elsen, ${ }^{67}$ R. C. Field, ${ }^{67}$ T. Glanzman, ${ }^{67}$ S. J. Gowdy, ${ }^{67}$

E. Grauges-Pous, ${ }^{67}$ T. Hadig, ${ }^{67}$ V. Halyo, ${ }^{67}$ T. Hryn'ova, ${ }^{67}$ W. R. Innes, ${ }^{67}$ C. P. Jessop, ${ }^{67}$ M. H. Kelsey, ${ }^{67}$ P. Kim, ${ }^{67}$

M. L. Kocian, ${ }^{67}$ U. Langenegger, ${ }^{67}$ D. W. G. S. Leith, ${ }^{67}$ J. Libby, ${ }^{67}$ S. Luitz, ${ }^{67}$ V. Luth, ${ }^{67}$ H. L. Lynch, ${ }^{67}$

H. Marsiske, ${ }^{67}$ R. Messner, ${ }^{67}$ D. R. Muller, ${ }^{67}$ C. P. O'Grady, ${ }^{67}$ V. E. Ozcan, ${ }^{67}$ A. Perazzo, ${ }^{67}$ M. Perl, ${ }^{67}$

S. Petrak, ${ }^{67}$ B. N. Ratcliff, ${ }^{67}$ A. Roodman, ${ }^{67}$ A. A. Salnikov, ${ }^{67}$ R. H. Schindler, ${ }^{67}$ J. Schwiening, ${ }^{67}$ G. Simi, ${ }^{67}$

A. Snyder ${ }^{67}$ A. Soha, ${ }^{67}$ J. Stelzer, ${ }^{67}$ D. Su, ${ }^{67}$ M. K. Sullivan, ${ }^{67}$ J. Va'vra, ${ }^{67}$ S. R. Wagner, ${ }^{67}$ M. Weaver, ${ }^{67}$

A. J. R. Weinstein, ${ }^{67}$ W. J. Wisniewski, ${ }^{67}$ D. H. Wright, ${ }^{67}$ C. C. Young, ${ }^{67}$ P. R. Burchat, ${ }^{68}$ A. J. Edwards, ${ }^{68}$

T. I. Meyer, ${ }^{68}$ B. A. Petersen, ${ }^{68}$ C. Roat, ${ }^{68}$ M. Ahmed, ${ }^{69}$ S. Ahmed, ${ }^{69}$ M. S. Alam, ${ }^{69}$ J. A. Ernst, ${ }^{69}$ M. A. Saeed, ${ }^{69}$

M. Saleem, ${ }^{69}$ F. R. Wappler, ${ }^{69}$ W. Bugg, ${ }^{70}$ M. Krishnamurthy, ${ }^{70}$ S. M. Spanier, ${ }^{70}$ R. Eckmann, ${ }^{71}$ H. Kim, ${ }^{71}$

J. L. Ritchie, ${ }^{71}$ R. F. Schwitters, ${ }^{71}$ J. M. Izen, ${ }^{72}$ I. Kitayama, ${ }^{72}$ X. C. Lou, ${ }^{72}$ S. Ye ${ }^{72}$ F. Bianchi, ${ }^{73}$ M. Bona, ${ }^{73}$

F. Gallo, ${ }^{73}$ D. Gamba, ${ }^{73}$ C. Borean, ${ }^{74}$ L. Bosisio, ${ }^{74}$ G. Della Ricca, ${ }^{74}$ S. Dittongo, ${ }^{74}$ S. Grancagnolo, ${ }^{74}$ L. Lanceri, ${ }^{74}$

P. Poropat,${ }^{74, \ddagger}$ L. Vitale, ${ }^{74}$ G. Vuagnin, ${ }^{74}$ R. S. Panvini, ${ }^{75}$ Sw. Banerjee, ${ }^{76}$ C. M. Brown, ${ }^{76}$ D. Fortin, ${ }^{76}$

P. D. Jackson, ${ }^{76}$ R. Kowalewski, ${ }^{76}$ J. M. Roney, ${ }^{76}$ H. R. Band, ${ }^{77}$ S. Dasu, ${ }^{77}$ M. Datta, ${ }^{77}$ A. M. Eichenbaum, ${ }^{77}$

J. R. Johnson, ${ }^{77}$ P. E. Kutter, ${ }^{77}$ H. Li, ${ }^{77}$ R. Liu, ${ }^{77}$ F. Di Lodovico, ${ }^{77}$ A. Mihalyi, ${ }^{77}$ A. K. Mohapatra, ${ }^{77}$ Y. Pan, ${ }^{77}$

R. Prepost, ${ }^{77}$ S. J. Sekula, ${ }^{77}$ J. H. von Wimmersperg-Toeller, ${ }^{77}$ J. Wu, ${ }^{77}$ S. L. Wu, ${ }^{77}$ Z. Yu, ${ }^{77}$ and H. Neal ${ }^{78}$

(The BABAR Collaboration)

${ }^{1}$ Laboratoire de Physique des Particules, F-74941 Annecy-le-Vieux, France

${ }^{2}$ Università di Bari, Dipartimento di Fisica and INFN, I-70126 Bari, Italy

${ }^{3}$ Institute of High Energy Physics, Beijing 100039, China

${ }^{4}$ University of Bergen, Inst. of Physics, N-5007 Bergen, Norway

${ }^{5}$ Lawrence Berkeley National Laboratory and University of California, Berkeley, CA 94720, USA

${ }^{6}$ University of Birmingham, Birmingham, B15 2TT, United Kingdom

${ }^{7}$ Ruhr Universität Bochum, Institut für Experimentalphysik 1, D-44780 Bochum, Germany

${ }^{8}$ University of Bristol, Bristol BS8 1TL, United Kingdom

${ }^{9}$ University of British Columbia, Vancouver, BC, Canada V6T $1 Z 1$

${ }^{10}$ Brunel University, Uxbridge, Middlesex UB8 3PH, United Kingdom

${ }^{11}$ Budker Institute of Nuclear Physics, Novosibirsk 630090, Russia

${ }^{12}$ University of California at Irvine, Irvine, CA 92697, USA

${ }^{13}$ University of California at Los Angeles, Los Angeles, CA 90024, USA

${ }^{14}$ University of California at Riverside, Riverside, CA 92521, USA

${ }^{15}$ University of California at San Diego, La Jolla, CA 92093, USA

${ }^{16}$ University of California at Santa Barbara, Santa Barbara, CA 93106, USA

${ }^{17}$ University of California at Santa Cruz, Institute for Particle Physics, Santa Cruz, CA 95064, USA 
${ }^{18}$ California Institute of Technology, Pasadena, CA 91125, USA

${ }^{19}$ University of Cincinnati, Cincinnati, $O H$ 45221, USA

${ }^{20}$ University of Colorado, Boulder, CO 80309, USA

${ }^{21}$ Colorado State University, Fort Collins, CO 80523, USA

${ }^{22}$ Technische Universität Dresden, Institut für Kern- und Teilchenphysik, D-01062 Dresden, Germany

${ }^{23}$ Ecole Polytechnique, LLR, F-91128 Palaiseau, France

${ }^{24}$ University of Edinburgh, Edinburgh EH9 3JZ, United Kingdom

${ }^{25}$ Università di Ferrara, Dipartimento di Fisica and INFN, I-44100 Ferrara, Italy

${ }^{26}$ Florida A\&M University, Tallahassee, FL 3230\%, USA

${ }^{27}$ Laboratori Nazionali di Frascati dell'INFN, I-00044 Frascati, Italy

${ }^{28}$ Università di Genova, Dipartimento di Fisica and INFN, I-16146 Genova, Italy

${ }^{29}$ Harvard University, Cambridge, MA 02138, USA

${ }^{30}$ Imperial College London, London, SW7 2BW, United Kingdom

${ }^{31}$ University of Iowa, Iowa City, IA 52242, USA

${ }^{32}$ Iowa State University, Ames, IA 50011-3160, USA

${ }^{33}$ Laboratoire de l'Accélérateur Linéaire, F-91898 Orsay, France

${ }^{34}$ Lawrence Livermore National Laboratory, Livermore, CA 94550, USA

${ }^{35}$ University of Liverpool, Liverpool L69 3BX, United Kingdom

${ }^{36}$ Queen Mary, University of London, E1 4NS, United Kingdom

${ }^{37}$ University of London, Royal Holloway and Bedford New College, Egham, Surrey TW20 0EX, United Kingdom

${ }^{38}$ University of Louisville, Louisville, KY 40292, USA

${ }^{39}$ University of Manchester, Manchester M13 9PL, United Kingdom

${ }^{40}$ University of Maryland, College Park, MD 20742, USA

${ }^{41}$ University of Massachusetts, Amherst, MA 01003, USA

${ }^{42}$ Massachusetts Institute of Technology, Laboratory for Nuclear Science, Cambridge, MA 02139, USA

${ }^{43}$ McGill University, Montréal, QC, Canada H3A $2 T 8$

${ }^{44}$ Università di Milano, Dipartimento di Fisica and INFN, I-20133 Milano, Italy

${ }^{45}$ University of Mississippi, University, MS 38677, USA

${ }^{46}$ Université de Montréal, Laboratoire René J. A. Lévesque, Montréal, QC, Canada H3C 3J7

${ }^{47}$ Mount Holyoke College, South Hadley, MA 01075, USA

${ }^{48}$ Università di Napoli Federico II, Dipartimento di Scienze Fisiche and INFN, I-80126, Napoli, Italy

${ }^{49}$ NIKHEF, National Institute for Nuclear Physics and High Energy Physics, NL-1009 DB Amsterdam, The Netherlands

${ }^{50}$ University of Notre Dame, Notre Dame, IN 46556, USA

${ }^{51}$ Oak Ridge National Laboratory, Oak Ridge, TN 37831, USA

${ }^{52}$ Ohio State University, Columbus, OH 43210, USA

${ }^{53}$ University of Oregon, Eugene, OR 97403, USA

${ }^{54}$ Università di Padova, Dipartimento di Fisica and INFN, I-35131 Padova, Italy

${ }^{55}$ Universités Paris VI et VII, Lab de Physique Nucléaire H. E., F-75252 Paris, France

${ }^{56}$ Università di Pavia, Dipartimento di Elettronica and INFN, I-27100 Pavia, Italy

${ }^{57}$ University of Pennsylvania, Philadelphia, PA 19104, USA

${ }^{58}$ Università di Perugia and INFN, I-06100 Perugia, Italy

${ }^{59}$ Università di Pisa, Dipartimento di Fisica, Scuola Normale Superiore and INFN, I-56127 Pisa, Italy

${ }^{60}$ Prairie View A\&M University, Prairie View, TX 77446, USA

${ }^{61}$ Princeton University, Princeton, NJ 08544, USA

${ }^{62}$ Università di Roma La Sapienza, Dipartimento di Fisica and INFN, I-00185 Roma, Italy

${ }^{63}$ Universität Rostock, D-18051 Rostock, Germany

${ }^{64}$ Rutherford Appleton Laboratory, Chilton, Didcot, Oxon, OX11 0QX, United Kingdom

${ }^{65}$ DSM/Dapnia, CEA/Saclay, F-91191 Gif-sur-Yvette, France

${ }^{66}$ University of South Carolina, Columbia, SC 29208, USA

${ }^{67}$ Stanford Linear Accelerator Center, Stanford, CA 94309, USA

${ }^{68}$ Stanford University, Stanford, CA 94305-4060, USA

${ }^{69}$ State Univ. of New York, Albany, NY 12222, USA

${ }^{70}$ University of Tennessee, Knoxville, TN 37996, USA

${ }^{71}$ University of Texas at Austin, Austin, TX 78712, USA

${ }^{72}$ University of Texas at Dallas, Richardson, TX 75083, USA

${ }^{73}$ Università di Torino, Dipartimento di Fisica Sperimentale and INFN, I-10125 Torino, Italy

${ }^{74}$ Università di Trieste, Dipartimento di Fisica and INFN, I-34127 Trieste, Italy

${ }^{75}$ Vanderbilt University, Nashville, TN 37235, USA

${ }^{76}$ University of Victoria, Victoria, BC, Canada V8W 3P6

${ }^{77}$ University of Wisconsin, Madison, WI 53706, USA

${ }^{78}$ Yale University, New Haven, CT 06511, USA

(Dated: December 19, 2003) 


\begin{abstract}
We present a measurement of the branching fraction for the decay $B^{-} \rightarrow D^{0} K^{*-}$ using a sample of approximately 86 million $B \bar{B}$ pairs collected by the BABAR detector from $e^{+} e^{-}$collisions near the $\Upsilon(4 S)$ resonance. The $D^{0}$ is detected through its decays to $K^{-} \pi^{+}, K^{-} \pi^{+} \pi^{0}$ and $K^{-} \pi^{+} \pi^{-} \pi^{+}$, and the $K^{*-}$ through its decay to $K_{S}^{0} \pi^{-}$. We measure the branching fraction to be $\mathcal{B}\left(B^{-} \rightarrow D^{0} K^{*-}\right)$ $=(6.3 \pm 0.7$ (stat. $) \pm 0.5$ (syst. $)) \times 10^{-4}$.

PACS numbers: $13.25 . \mathrm{Hw} 14.40 . \mathrm{Nd}$
\end{abstract}

A comprehensive test of $C P$ violation within the Standard Model requires precision measurements of the three sides and three angles of the Unitarity Triangle, which are combinations of various Cabibbo-KobayashiMaskawa (CKM) matrix elements [? ]. The measurement of the angle $\gamma$ of the Unitarity Triangle is challenging and requires larger samples of $B$ mesons than are currently available. A precise determination of $\gamma$ at the $B$ factories is likely to use many different decay modes. Decays of the form $B \rightarrow D^{(*)} K^{(*)}$ can provide a theoretically clean determination of $\gamma[?]$. For some of the proposed methods, there are distinct advantages to using the $\mathrm{K}^{*}$ modes [? ]. In this paper, we measure the branching fraction for one of these decays, $B^{-} \rightarrow D^{0} K^{*-}[?]$, which was first observed by the CLEO experiment [?]. If the $D^{0}$ is reconstructed in its decay to $C P$ eigenstates, the $b \rightarrow c \bar{u} s$ and $b \rightarrow u \bar{c} s$ quark transitions interfere, giving access to the phase $\gamma$ through the measurement of direct $C P$ violation asymmetries. However, the branching fractions for $D^{0}$ decays to $C P$ eigenstates are only of the order of $1 \%$, too small for the size of the available data sample. Therefore, for this analysis, we use decay modes of the $D^{0}$ and $K^{*-}$ that have clear experimental signatures and sufficiently high branching fractions. This measurement provides an important first step towards establishing the feasibility of using the decay $B^{-} \rightarrow D^{0} K^{*-}$ for a future determination of $\gamma$.

We present here a measurement of the branching fraction for the decay $B^{-} \rightarrow D^{0} K^{*-}$ using data collected with the BABAR detector at the PEP-II $e^{+} e^{-}$storage ring. The data correspond to an integrated luminosity of $81.5 \mathrm{fb}^{-1}$ taken at center-of-mass energies close to the $\Upsilon(4 S)$ resonance, giving a sample of approximately 86 million $B \bar{B}$ pairs. We reconstruct $D^{0}$ candidates through the decays $D^{0} \rightarrow K^{-} \pi^{+}, D^{0} \rightarrow K^{-} \pi^{+} \pi^{0}$ and $D^{0} \rightarrow K^{-} \pi^{+} \pi^{-} \pi^{+}$. $K^{*-}$ candidates are identified through the decay $K^{*-} \rightarrow K_{S}^{0} \pi^{-}$, with the $K_{S}^{0}$ decaying to a pair of charged pions.

A detailed description of the BABAR detector can be found elsewhere [? ]. Only detector components relevant to this analysis are described here. Charged-particle trajectories are measured by a five-layer double-sided silicon vertex tracker (SVT) and a 40-layer drift chamber $(\mathrm{DCH})$, operating in the field of a $1.5-\mathrm{T}$ solenoid.

\footnotetext{
*Also with Università della Basilicata, Potenza, Italy

${ }^{\dagger}$ Also with IFIC, Instituto de Física Corpuscular, CSICUniversidad de Valencia, Valencia, Spain

$\ddagger$ Deceased
}

Charged-particle identification is achieved by combining measurements of ionization energy loss $(\mathrm{d} E / \mathrm{d} x)$ in the $\mathrm{DCH}$ and SVT with information from a detector of internally reflected Cherenkov light (DIRC). Photons are detected in a $\mathrm{CsI}(\mathrm{Tl})$ electromagnetic calorimeter (EMC).

We set the event-selection criteria to minimize the statistical error on the branching fraction, using simulations of the signal and background. In general, charged tracks are required to have at least $12 \mathrm{DCH}$ hits and a minimum transverse momentum of $0.1 \mathrm{GeV}$, and to originate from the interaction point, within $10 \mathrm{~cm}$ along the beam direction and $1.5 \mathrm{~cm}$ in the transverse plane. We use less restrictive selection criteria for tracks used to reconstruct $K_{S}^{0} \rightarrow \pi^{+} \pi^{-}$candidates, to allow for displaced $K_{S}^{0}$ decay vertices. Photon candidates are identified in the EMC as deposits of energy isolated from charged tracks. They are required to have a minimum energy of $30 \mathrm{MeV}$ and a shower shape consistent with that of a photon.

We use pairs of photons to reconstruct $\pi^{0}$ candidates, which are required to have an invariant mass between 125 and $144 \mathrm{MeV}$. We reconstruct $K_{S}^{0}$ candidates from pairs of oppositely charged tracks fitted to a common vertex. They are required to have an invariant mass within $8 \mathrm{MeV}$ of the $K_{S}^{0}$ mass [? ].

To reconstruct $K^{*-}$ candidates, we combine $K_{S}^{0}$ candidates with charged tracks. We require the $K^{*-}$ candidate to have an invariant mass within $75 \mathrm{MeV}$ of $892 \mathrm{MeV}$. In addition, the $K_{S}^{0}$ vertex is required to be displaced by at least $3 \mathrm{~mm}$ from the $K^{*-}$ vertex.

We reconstruct $D^{0}$ candidates from the appropriate combination of tracks and $\pi^{0}$ candidates. The $K^{-}$tracks must satisfy kaon identification criteria resulting in an efficiency of $80 \%-95 \%$ depending on the momentum. The probability of a pion to be misidentified as a kaon is less than $5 \%$. We require the momenta of the $K^{-}$candidates to be greater than $250 \mathrm{MeV}$ and their polar angle (relative to magnetic-field axis) to be in the interval $0.25<\theta<$ $2.55 \mathrm{rad}$ to restrict them to a fiducial region where the kaon identification performance can be determined with small uncertainty. The tracks from the $D^{0}$ are fitted to a common vertex and we accept candidates if they have an invariant mass within 18 (14) $\mathrm{MeV}$ of the $D^{0}$ mass for the $K^{-} \pi^{+}\left(K^{-} \pi^{+} \pi^{-} \pi^{+}\right)$decay. For the $K^{-} \pi^{+} \pi^{0}$ decay, we use an asymmetric mass requirement $-29<(m-$ $1865 \mathrm{MeV})<+24 \mathrm{MeV}$, reflecting the distribution of the energy of the photons from the $\pi^{0}$ decay. It is known that the decay $D^{0} \rightarrow K^{-} \pi^{+} \pi^{0}$ occurs predominantly through an intermediate state $\left(K^{*-}(892)\right.$ or $\left.\rho^{+}(770)\right)$. Hence, to reduce the combinatorial background in the $K^{-} \pi^{+} \pi^{0}$ decay, we select events in the enhanced regions of the 
Dalitz plot, using amplitudes and phases determined by the CLEO experiment [? ].

In reconstructing the decay chain, the measured momentum vector of each intermediate particle is determined by refitting the momenta of its decay products, constraining the mass to the nominal mass of the particle and requiring the decay products to originate from a common point. For the $K^{*-}$ resonance only a geometrical constraint is used in this kinematic fit. Finally, to reconstruct $B^{-}$decays, $D^{0}$ candidates are combined with $K^{*-}$ candidates.

The dominant background is from $e^{+} e^{-} \rightarrow q \bar{q}$ production. We suppress this background using requirements on the event topology and kinematics, and through the use of a Fisher discriminant. The ratio of the second and zeroth Fox-Wolfram moments [? ], which is a measure of the event sphericity and is close to zero for approximately spherical events, is required to be less than 0.5 . The absolute value of the cosine of the angle between the thrust axis of the $B$ candidate and the thrust axis of the rest of the event, $\left|\cos \theta_{T}\right|$, is peaked at one for continuum events and is approximately flat for $B$ decays. We require $\left|\cos \theta_{T}\right|<0.8$ for $K^{-} \pi^{+}$and $K^{-} \pi^{+} \pi^{0}$ decays and $\left|\cos \theta_{T}\right|<0.75$ for $K^{-} \pi^{+} \pi^{-} \pi^{+}$decays. The Fisher discriminant is built from the momentum of all particles in the event (excluding those used to form the $B$ candidate) and the angle between this momentum and the thrust axis of the reconstructed $B^{-}$, both in the centerof-mass frame [? ]. The $K^{*-}$ helicity angle, $\theta_{H}$, defined as the angle between the $\pi^{-}$from the $K^{*-}$ decay and the $B^{-}$flight direction in the rest frame of the $K^{*-}$, follows a $\cos ^{2} \theta_{H}$ distribution for signal events and is approximately flat for continuum events. To further reject continuum background in the $K^{-} \pi^{+} \pi^{-} \pi^{+}$channel, we require $\left|\cos \theta_{H}\right|>0.4$.

The selection criteria just described reject all but approximately $0.001 \%$ of the background, while retaining between $4 \%$ and $13 \%$ of the signal, depending on the $D^{0}$ mode. In the case of events with more than one $B^{-}$candidate (5-17\%, depending on the $D^{0}$ mode), we choose the best candidate on the basis of the $\chi^{2}$ formed from the differences of the measured and true $B^{-}, D^{0}$, and $K_{S}^{0}$ masses, scaled by the mass resolutions. Studies of simulated signal events have determined that the algorithm does not introduce a bias and chooses the correct $B^{-}$candidate in approximately $80 \%$ of the events with multiple candidates.

Finally, we identify $B$-meson decays kinematically using two nearly independent variables: the energysubstituted $B$ mass $m_{\mathrm{ES}}=\sqrt{\left(s / 2+\mathbf{p}_{\mathbf{0}} \cdot \mathbf{p}_{\mathbf{B}}\right)^{2} / E_{0}^{2}-p_{B}^{2}}$, where the subscripts 0 and $B$ refer to the $e^{+} e^{-}$system and the $B$ candidate respectively, $s$ is the square of the center-of-mass energy, and energies $(E)$ and momentum vectors $(\mathbf{p})$ are computed in the laboratory frame; and $\Delta E=E_{B}^{*}-\sqrt{s} / 2$, where $E_{B}^{*}$ is the $B$ candidate energy in the center-of-mass frame. We select $B^{-}$candidates with $|\Delta E|<25 \mathrm{MeV}$, which corresponds to approximately $\pm 2.2 \sigma$ (where the resolution $\sigma$ is found to be independent of the $D^{0}$ decay mode). In addition, the signal events are expected to have values of $m_{\mathrm{ES}}$ close to the $B^{-}$mass.

We determine the signal yield of $B^{-} \rightarrow D^{0} K^{*-}$ events by performing an unbinned maximum likelihood fit to the $m_{\mathrm{ES}}$ distribution of the selected candidates for the signal region in $\Delta E$. The signal distribution is parameterized as a Gaussian function and the combinatorial background as a threshold function [? ]. All parameters except the endpoint of the threshold function are unconstrained in the fit.

The signal yield determined from the fit potentially includes backgrounds from other $B \bar{B}$ decays that also peak in $m_{\mathrm{ES}}$. To investigate this, we have studied a simulated sample of generic $B \bar{B}$ decays and also high statistics simulated samples of other $B \rightarrow D^{(*)} K^{(*)}$ decays. The simulation indicates no enhancement in the signal region from this background. Therefore, we assume that the peaking background is negligible and the uncertainty in its determination from the studies of various simulated event samples is included as a systematic error. We have also verified that use of the $B^{-}$mass and error in the $\chi^{2}$ calculation for the choice of the best $B^{-}$candidate does not affect the smooth shape of the background in $m_{\mathrm{ES}}$.

Figure ?? shows the $m_{\mathrm{ES}}$ distribution for the three different $D^{0}$ decay modes with the fit function superimposed. A clear signal is seen in all cases. The signal yield and the size of the combinatorial background in the signal region, i.e., for events with $m_{\mathrm{ES}}>5.270 \mathrm{GeV}$, are detailed in Table ??. We observe a total of $161 \pm 17$ $B^{-} \rightarrow D^{0} K^{*-}$ events. We have studied the $\cos \theta_{H}$ distribution for the selected candidates and determined that the data are consistent with pure $B^{-} \rightarrow D^{0} K^{*-}$ decay.

TABLE I: Signal yield, number of background events, and efficiency for the three $D^{0}$ decay modes in the signal region $\left(m_{\mathrm{ES}}>5.270 \mathrm{GeV}\right)$. Yields are extracted from the fits to the $m_{\mathrm{ES}}$ distribution from data (errors are statistical only). Efficiencies are computed from simulated events.

\begin{tabular}{lccc}
\hline \hline & $K^{-} \pi^{+}$ & $K^{-} \pi^{+} \pi^{0}$ & $K^{-} \pi^{+} \pi^{-} \pi^{+}$ \\
\hline Signal Yield & $56.2 \pm 9.4$ & $51.7 \pm 11.0$ & $52.6 \pm 8.7$ \\
Background & $19.5 \pm 4.3$ & $37.7 \pm 6.2$ & $16.4 \pm 3.6$ \\
\hline Efficiency (\%) & 12.8 & 3.5 & 4.0 \\
\hline \hline
\end{tabular}

We determine the selection efficiency for each sample of $B^{-} \rightarrow D^{0} K^{*-}$ events from samples of simulated signal events. We apply small corrections determined from data to the efficiency calculation to account for the overestimation of the tracking and particle-identification performance, and of the $\pi^{0}$ and $K_{S}^{0}$ reconstruction efficiencies in the Monte Carlo simulation. The product of these efficiency corrections is about 0.9 .

To quantify the ability of the simulation to model the variables used in the event selection, we use a sample of $B^{-} \rightarrow D^{0} \pi^{-}$events from data and Monte Carlo simulation. This sample is kinematically similar to the $B^{-} \rightarrow D^{0} K^{*-}$ decay. We select $B^{-} \rightarrow D^{0} \pi^{-}$events in 


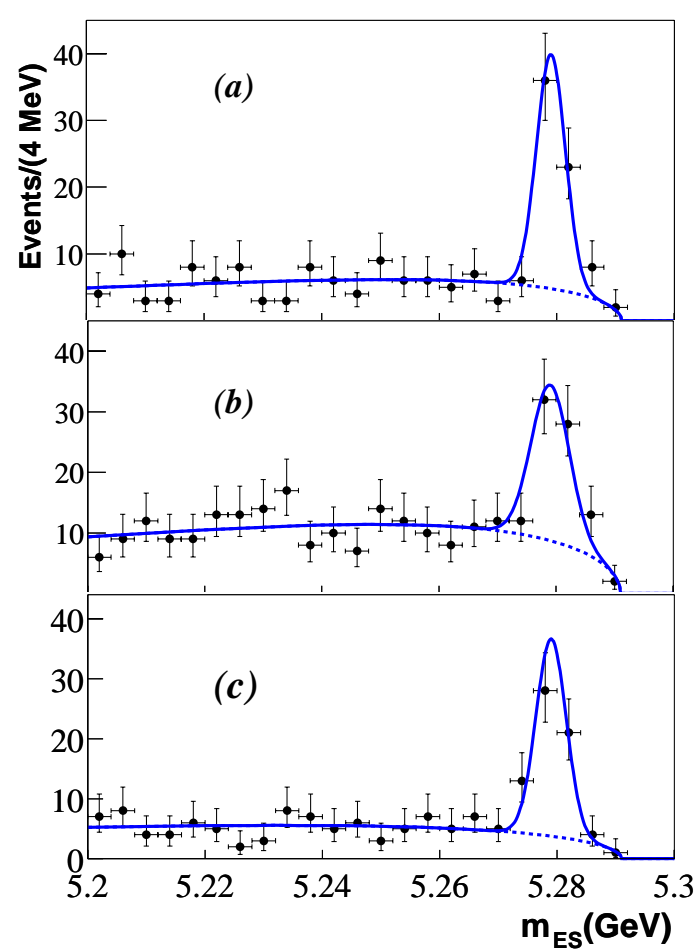

FIG. 1: The $m_{\mathrm{ES}}$ distributions of $B^{-} \rightarrow D^{0} K^{*-}$ candidates: (a) $D^{0} \rightarrow K^{-} \pi^{+}$, (b) $D^{0} \rightarrow K^{-} \pi^{+} \pi^{0}$, and (c) $D^{0} \rightarrow K^{-} \pi^{+} \pi^{-} \pi^{+}$. The solid lines show the fit used to extract the signal yields, with the distribution parameterized as a Gaussian plus a threshold function as described in the text. The dashed line indicates the combinatorial background component.

the same way as the $B^{-} \rightarrow D^{0} K^{*-}$ sample, with the additional requirement that the $\pi^{-}$fails loose kaon identification criteria, to remove $B^{-} \rightarrow D^{0} K^{-}$events. Approximately $3000 B^{-} \rightarrow D^{0} \pi^{-}$candidates in each $D^{0}$ decay mode are selected from the data. The purity of the sample is $94 \%$ for the $K^{-} \pi^{+} \pi^{0}$ decay and $98 \%$ for the $K^{-} \pi^{+}$and $K^{-} \pi^{+} \pi^{-} \pi^{+}$decays. We use this sample to determine correction factors for the efficiencies for the $B^{-} \rightarrow D^{0} K^{*-}$ selection. The obtained correction factors vary from about 0.95 for the $K^{-} \pi^{+}$and $K^{-} \pi^{+} \pi^{-} \pi^{+}$decays to 0.85 for the $K^{-} \pi^{+} \pi^{0}$ decay. We include the statistical precision of these corrections in the systematic error of the branching fraction. The selection efficiency after all corrections are reported in Table ??.

We determine the branching fraction separately for each of the $D^{0}$ decay modes from:

$$
\mathcal{B}\left(B^{-} \rightarrow D^{0} K^{*-}\right)=\frac{N}{\epsilon \cdot N_{B \bar{B}} \cdot \mathcal{B}_{D^{0}} \cdot \mathcal{B}_{K^{*-}} \cdot \mathcal{B}_{K_{S}^{0}} \cdot \mathcal{B}_{\pi^{0}}}
$$

for a signal yield of $N$ events, an efficiency $\epsilon$ and a sample containing $N_{B \bar{B}}$ pairs of $B$ mesons. $\mathcal{B}_{D^{0}}, \mathcal{B}_{K^{*-}}, \mathcal{B}_{K_{S}^{0}}$ and $\mathcal{B}_{\pi^{0}}$, the branching fractions for the $D^{0}, K^{*-}, K_{S}^{0}$ and $\pi^{0}$ respectively to the relevant final states, are obtained from Ref. [? ] $\left(\mathcal{B}_{\pi^{0}}\right.$ in equation is only relevant for the $K^{-} \pi^{+} \pi^{0}$ mode). We assume that the $\Upsilon(4 S)$ decays to pairs of $B^{+} B^{-}$and $B^{0} \bar{B}^{0}$ mesons with equal probability and we do not include any additional uncertainty due to this assumption.

We have identified several sources of systematic uncertainty as significant. The number of $B \bar{B}$ pairs in the data sample is known with an uncertainty of $1.1 \%$. The uncertainties in the $D^{0}$ branching ratios are taken from Ref. [? ]. We determine the systematic errors arising from uncertainties in track, $K_{S}^{0}$ and $\pi^{0}$ reconstruction and in kaon identification from studies of high statistics data control samples. The uncertainty in the track reconstruction efficiency is determined to be $0.8 \%$ per track originating from the interaction region. There is an additional uncertainty of $3 \%$ arising from the knowledge of the $K_{S}^{0}$ reconstruction efficiency. The charged kaon iden-

TABLE II: Systematic uncertainty estimates for each of the three $D^{0}$ decay samples.

\begin{tabular}{lccc}
\hline \hline Source & \multicolumn{3}{c}{ Uncertainty (\%) } \\
\hline & $K^{-} \pi^{+}$ & $K^{-} \pi^{+} \pi^{0}$ & $K^{-} \pi^{+} \pi^{-} \pi^{+}$ \\
\hline Number of $B \bar{B}$ events & 1.1 & 1.1 & 1.1 \\
Simulation statistics & 5.6 & 6.5 & 6.0 \\
$D^{0}$ branching ratio & 2.4 & 6.2 & 4.2 \\
Tracking efficiency & 2.4 & 2.4 & 4.0 \\
$K_{S}^{0}$ efficiency & 3.0 & 3.0 & 3.0 \\
Particle identification & 2.0 & 2.0 & 2.0 \\
$\pi^{0}$ efficiency & - & 5.0 & - \\
Peaking background & 2.3 & 1.4 & 3.1 \\
$K^{*-}$ lineshape & 3.0 & 3.0 & 3.0 \\
Data/simulation differences & 1.4 & 2.4 & 2.1 \\
\hline Total & 8.6 & 11.9 & 10.3 \\
\hline \hline
\end{tabular}

tification leads to a systematic uncertainty of $2 \%$, and the $\pi^{0}$ reconstruction to a systematic uncertainty of $5 \%$. The systematic error from the knowledge of the peaking background is taken from the studies of various simulated data samples described above. An additional uncertainty from the knowledge of the $K^{*-}$ lineshape has been determined to be $3 \%$. Finally, we include the errors on the correction factors determined from the $B^{-} \rightarrow D^{0} \pi^{-}$sample. We have studied the uncertainty in the parameterization of the background and of the signal by repeating the $m_{\mathrm{ES}}$ fits with different combinations of parameters of the functional form fixed to values obtained either from simulation or from studies of sideband regions in $\Delta E$. We conclude that the systematic uncertainty from this source is negligible. A summary of the systematic errors estimate is shown in Table ??.

The resulting $B$ branching fractions corresponding to three different $D^{0}$ decay modes are listed in Table ?? We determine the weighted average of the three measurements, $\mathcal{B}\left(B^{-} \rightarrow D^{0} K^{*-}\right)=(6.3 \pm 0.7 \pm 0.5) \times 10^{-4}$, taking into account the correlations between the systematic uncertainties. The result of this analysis is in 
TABLE III: Measured branching fraction $\mathcal{B}\left(B^{-} \rightarrow D^{0} K^{*-}\right)$. The first errors are statistical and the second systematic.

\begin{tabular}{lc}
\hline \hline Decay Mode & $\mathcal{B}\left(10^{-4}\right)$ \\
\hline$D^{0} \rightarrow K^{-} \pi^{+}$ & $5.8 \pm 1.0 \pm 0.5$ \\
$D^{0} \rightarrow K^{-} \pi^{+} \pi^{0}$ & $5.8 \pm 1.2 \pm 0.7$ \\
$D^{0} \rightarrow K^{-} \pi^{+} \pi^{-} \pi^{+}$ & $8.7 \pm 1.5 \pm 0.9$ \\
\hline Weighted Average & $6.3 \pm 0.7 \pm 0.5$ \\
\hline \hline
\end{tabular}

good agreement with a previous measurement by CLEO, $\mathcal{B}\left(B^{-} \rightarrow D^{0} K^{*-}\right)=(6.1 \pm 1.6 \pm 1.7) \times 10^{-4}[?]$.

In summary, we have studied the decay $B^{-} \rightarrow D^{0} K^{*-}$, where the $D^{0}$ was detected through its decays to $K^{-} \pi^{+}$, $K^{-} \pi^{+} \pi^{0}$ and $K^{-} \pi^{+} \pi^{-} \pi^{+}$and the $K^{*-}$ through its decay to $K_{S}^{0} \pi^{-}$. We have measured the branching fraction $\mathcal{B}\left(B^{-} \rightarrow D^{0} K^{*-}\right)=(6.3 \pm 0.7 \pm 0.5) \times 10^{-4}$. This is in good agreement with the previous measurement of this branching fraction, and significantly improves on its pre- cision. In the future, with larger data samples, this decay will be studied with the $D^{0}$ reconstructed in $C P$ eigenstates. Eventually it is hoped that decays of the form $B \rightarrow D^{(*)} K^{(*)}$ can provide important constraints on the angle $\gamma$ of the Unitarity Triangle.

We are grateful for the excellent luminosity and machine conditions provided by our PEP-II colleagues, and for the substantial dedicated effort from the computing organizations that support BABAR. The collaborating institutions wish to thank SLAC for its support and kind hospitality. This work is supported by DOE and NSF (USA), NSERC (Canada), IHEP (China), CEA and CNRS-IN2P3 (France), BMBF and DFG (Germany), INFN (Italy), FOM (The Netherlands), NFR (Norway), MIST (Russia), and PPARC (United Kingdom). Individuals have received support from the A. P. Sloan Foundation, Research Corporation, and Alexander von Humboldt Foundation.
[] N. Cabibbo, Phys. Rev. Lett. 10, 531 (1963); M. Kobayashi and T. Maskawa, Prog. Theor. Phys. 49, 652 (1973).

[] M. Gronau and D. Wyler, Phys. Lett. B 265, 172 (1991); I. Dunietz, Phys. Lett. B 270, 75 (1991); D. Atwood, G. Eilam, M. Gronau and A. Soni, Phys. Lett. B 341, 372 (1995); D. Atwood, I. Dunietz and A. Soni, Phys. Rev. Lett. 78, 3257 (1997).

[] J. H. Jang and P. Ko, Phys. Rev. D 58, 111302 (1998); M. Gronau and J. L. Rosner, Phys. Lett. B 439, 171 (1998).

[] Charge conjugate decays are implied throughout this paper.

[] CLEO Collaboration, R. Mahapatra et al., Phys. Rev. Lett. 88, 101803 (2002).
[] BABAR Collaboration, B.Aubert et al., Nucl. Instr. Meth. A 479, 1 (2002).

[] Particle Data Group, K. Hagiwara et al., Phys. Rev. D 66, 010001 (2002) and 2003 off-year partial update for the 2004 edition available on the PDG WWW pages (http://pdg.lbl.gov).

[] CLEO Collaboration, S.Kopp et al., Phys. Rev. D 63, 092001 (2001).

[] G. Fox and S. Wolfram, Phys. Rev. Lett. 41, 1581 (1978).

[] BABAR Collaboration, B. Aubert et al., Phys. Rev. Lett. 89, 281802 (2002).

[] ARGUS Collaboration, H. Albrecht et al., Phys. Lett. B 185, 218 (1987); ibid. 241, 278 (1990). 